snare group in SMSA level 1(18.51[SD:8.26]seconds vs. 23.84 [SD:15.07] seconds, $P=0.013)$ and in SMSA level $2(25.03$ [SD:15.32] seconds vs. 29.15[SD:24.82] seconds, $P=0.009$ ), respectively. (figure 1 )

Conclusions Colorectal polyps could be removed more efficient by using rotary snares than traditional snares in SMSA level 1 and SMSA level 2.

\section{IDDF2019-ABS-0054 PREVALENCE OF PERIAMPULLARY DIVERTICULUM AND ITS ASSOCIATION WITH PANCREATOBILIARY DISEASE IN PATIENTS WHO UNDERGO ENDOSCOPIC RETROGRADE CHOLANGIO PANCREATOGRAM (ERCP): A SINGLE CENTER EXPERIENCE IN SRI LANKA}

Karthiha Balendran*, Meththananda Nawarathne, Vadivelu Vijitharan, Sharafaath Shariff. National Hospital of Sri Lanka, Sri Lanka

\subsection{6/gutjnl-2019-IDDFabstracts. 128}

Background Periampullary diverticulum is an outpouching of the duodenum arising within the radius of $2-3 \mathrm{~cm}$ from the ampulla of Vater. It is usually asymptomatic and discovered incidentally during ERCP. The prevalence of this finding widely varies from $1 \%$ to $32.8 \%$ and its association with pathological conditions has been recognized in various studies. The aim of this study was to evaluate the prevalence of periampullary diverticulum and its association with pancreatobiliary diseases in the studied population.

Methods The study group of 2350 patients who underwent ERCP between 2014 and 2018 at the gastroenterology unit of the National Hospital of Sri Lanka was analyzed. The prevalence of patients with periampullary diverticulum was calculated. The demographic features and its association with pancreatobiliary diseases were analyzed in patients with periampullary diverticulum.

Results 132 patients with periampullary diverticulum with a mean age of 62 (range 25-89) were identified. Out of 132 patients, $72 \%$ were males. The prevalence rate of periampullary diverticulum was $5.6 \%$ in the studied population. Successful biliary or pancreatic cannulation was achieved in $97 \%$ of patients with periampullary diverticulum. The presence of choledocholithiasis was diagnosed in $73(55.3 \%)$ patients. $13(9.8 \%)$ patients had benign common bile duct stricture and cholangiocarcinoma was found in $11(8.3 \%)$ patients. In addition, a small number of patients had chronic calcified pancreatitis $(6,4.5 \%)$ choledochal cyst $(4,3 \%)$ pancreatic carcinoma $(4,3 \%)$ and ampullary neoplasm (3, 2.3\%). However, 14 (10.6\%) patients had no structural lesion as a cause for biliary obstruction.

Conclusions The prevalence of periampullary diverticulum in our study population is lower than most previous reports. The presence of periampullary diverticulum is found more commonly in the elderly population with male preponderance. A significant association between choledocholithiasis and periampullary diverticulum is observed in this study

\section{IDDF2019-ABS-0055 WHAT IS THE CAUSE OF THIS PORTAL HYPERTENSION CASE?}

RuiTing Ma*. The General Hospital of NinXia Medical University, Yinchuan, China
Background The most common diseases that cause isolated gastric varices (IGV) are pancreatic diseases, such as pancreatic cancer, pseudocyst, or pancreatitis. In this case, surgical pathology eventually proved to be pancreatic cancer.

Methods A 56-year old man who presented intermittent abdominal pain and abdominal distension was admitted to our hospital. The pain was located in the upper abdomen. These symptoms persist for about a year and become

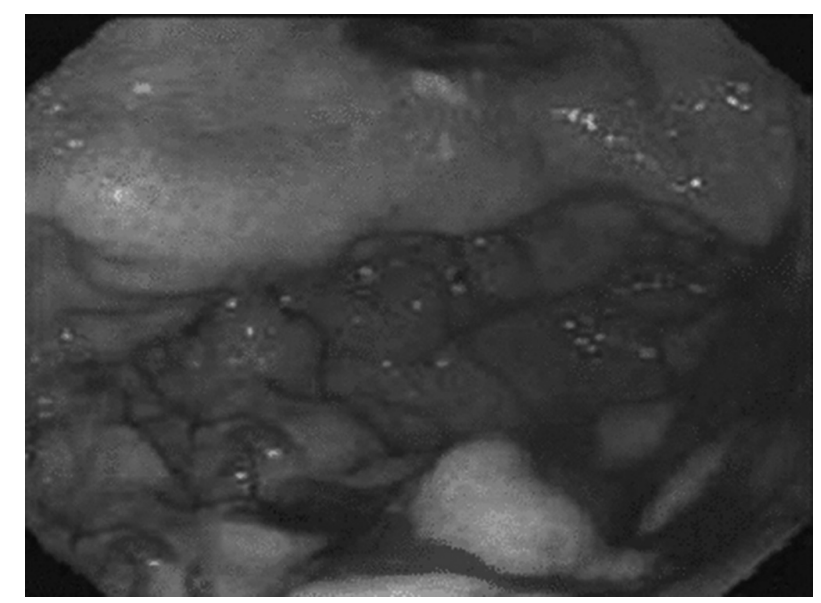

Abstract IDDF2019-ABS-0055 Figure 1

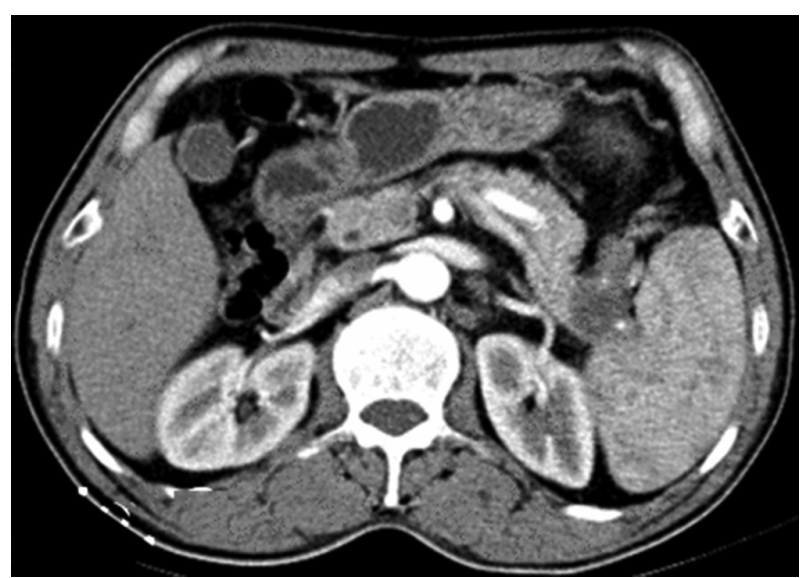

Abstract IDDF2019-ABS-0055 Figure 2

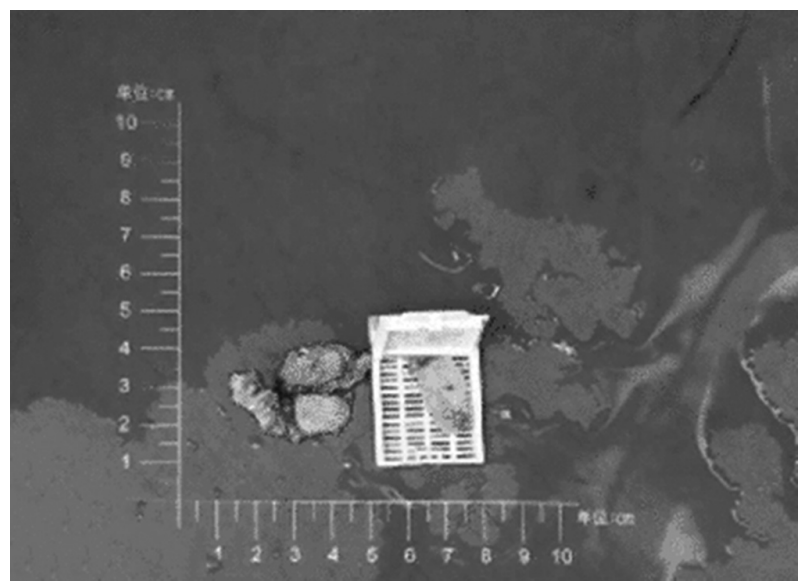

Abstract IDDF2019-ABS-0055 Figure 3 